\title{
Hemofilias e Doença de von Willebrand: uma revisão de literatura
}

\author{
Hemophilia and von Willebrand disease: review of literature
}

\author{
La hemofilia y la enfermedad de von Willibrand: una revisión de la literatura \\ Yago Tavares PINHEIRO ${ }^{1}$ \\ Elisa Caroline Leandro DA SILVA \\ Mônica Alves MACIEL ${ }^{2}$ \\ Emerson Tavares de SOUSA ${ }^{3}$ \\ ${ }^{\text {I} F i s i o t e r a p e u t a s . ~ F a c u l d a d e ~ M a u r i ́ c i o ~ d e ~ N a s s a u ~-~ F M N, ~ 58030-000 ~ J o a ̃ o ~ P e s s o a ~-~ P B, ~ B r a s i l ~}$ \\ ${ }^{2}$ Acadêmica de Nutrição. Universidade Federal da Paraíba - UFPB, 58051-900 João Pessoa - PB, Brasil \\ ${ }^{3}$ Mestre em Odontologia. Universidade Estadual de Campinas - UNICAMP, 13414-018 Piracicaba - SP, Brasil
}

\begin{abstract}
Resumo
A lesão de um tecido é o fator primário gerador do rompimento da continuidade do endotélio, seja por causa acidental ou provocada. Assim, as técnicas usadas pelos cirurgiões-dentistas estão associadas à hemorragia que podem sofrer um aumento decorrente de distúrbios hereditários da coagulação. Sendo assim, objetivou-se nesse estudo fazer um levantamento científico na literatura atual sobre as peculiaridades de cada tipo de hemofilia e da doença de Von Willebrand, evidenciando aspectos inerentes ao diagnóstico clinico e laboratorial, sempre numa perspectiva fisiológica. O presente estudo trata-se de uma revisão da literatura, com artigos em inglês e português, indexados nas bases de dados MedLine, Scielo e Lilacs e publicados entre os anos de 2001 a 2014. Para a busca dos artigos foram utilizados os seguintes descritores: coagulopatias hereditárias (hereditary coagulopathies), hemofilia (hemophilia) e doença de Von Willebrand (Von Willebrand disease). É de suma importância os profissionais terem conhecimento acerca do conceito, fisiopatologia e, principalmente, do diagnóstico das coagulopatias hemorrágicas, uma vez que a semelhança da apresentação clínica dessas afecções pode dificultar esse diagnóstico, interferindo, assim, no estabelecimento de um tratamento. Apesar desse estudo ter sido amplo, percebe-se a necessidade de mais evidências mais profundas acerca das coagulopatias hemorrágicas apresentadas.
\end{abstract}

Descritores: Transtornos da Coagulação do Sangue; Transtornos Hemorrágicos; Doenças de von Willebrand.

\begin{abstract}
The injury of a tissue is the primary disruption of generating factor of continuity of the endothelium, or by accidental causes or provocada. Thus, the techniques used by dentists are associated with bleeding which can be increased due to hereditary coagulation disorders. Therefore, this study aimed to make a scientific survey on the current literature on the characteristics of each type of hemophilia and Von Willebrand's disease, highlighting aspects inherent to the clinical and laboratory diagnosis, always a physiological perspective. This study it is a literature review, with articles in English and Portuguese, indexed in MedLine, Scielo and Lilacs and published between the years 2001 to 2014. To search for articles the following keywords were used: Hereditary coagulopathies (hereditary coagulopathies), hemophilia (hemophilia) and Von Willebrand disease (Von Willebrand disease). It is extremely important professionals have knowledge about the concept, pathophysiology and especially the diagnosis of hemorrhagic coagulopathy, since the similarity of the clinical presentation of these disorders can hinder the diagnosis, thus interfering in the establishment of a treatment. Although this study was broad, one sees the need for deeper evidence about the hemorrhagic coagulopathy presented.

Descriptors: Blood Coagulation Disorders; Hemorrhagic Disorders; von Willebrand Diseases.
\end{abstract}

\section{Resumen}

La lesión de un tejido es el factor principal que genera la ruptura de la continuidad del endotelio, ya sea por causas accidentales o intencionales. Por lo tanto, las técnicas utilizadas por los dentistas están asociados con una hemorragia que puede ser incrementado debido a trastornos de la coagulación hereditarios. Por lo tanto, este estudio tuvo como objetivo hacer un estudio científico de la literatura actual sobre las peculiaridades de cada tipo de hemofilia y la enfermedad de von Willebrand, destacando los aspectos del diagnóstico clínico y de laboratorio, siempre un punto de vista fisiológico. Este estudio trata de una revisión de la literatura, con artículos en Inglés y portugués, en Medline, Scielo y Lilacs y publicada entre los años 2001 a 2014. Para la búsqueda de los artículos se utilizaron los siguientes descriptores: coagulopatías hereditarias (coagulopatías hereditarias), hemofilia (hemofilia) y la enfermedad de von Willebrand (enfermedad de Von Willebrand). Es muy importante profesionales de conocimiento sobre el concepto, fisiopatología y especialmente el diagnóstico de coagulopatías hemorrágicas, ya que la similitud de la presentación clínica de estos trastornos puede dificultar el diagnóstico, lo que interfiere en el establecimiento de un tratamiento. Aunque este estudio era amplio, se percibe la necesidad de pruebas más profundas sobre las coagulopatías hemorrágicas presentadas.

Descriptores: Trastornos de la Coagulación Sanguínea; Trastornos Hemorrágicos; Enfermedades de von Willebrand.

\section{INTRODUÇÃO}

A lesão de um tecido é o fator primário gerador do rompimento da continuidade do endotélio, seja por causa acidental ou provocada ${ }^{1,2}$. Assim, as técnicas usadas pelos cirurgiões-dentistas estão associadas à hemorragia pósoperatória, a qual, na maioria dos casos, é autolimitada. Porém, existe uma pequena probabilidade de que haja um aumento da hemorragia devido a doenças hemorrágicas de caráter hereditário.

Os distúrbios hereditários da coagulação resultam de deficiências na estrutura e na quantidade de uma ou mais proteínas plasmáticas ${ }^{4}$. Pessoas acometidas por esse tipo de doença podem apresentar sangramento de gravidade variável ao nascimento ou que são diagnosticados ocasionalmente em pré-operatório ou exame periódico ${ }^{5,6}$.

Dentre as coagulopatias hereditárias as hemofilias e a doença de von Willebrand (DvW) são as mais comuns, sendo, portanto nosso objeto de estudo. Sendo assim, objetivou-se nesse estudo fazer um levantamento científico na literatura atual sobre as peculiaridades de cada tipo de hemofilia e da doença de Von Willebrand, evidenciando aspectos inerentes ao diagnóstico clinico e laboratorial, sempre numa perspectiva fisiológica. 


\section{MATERIAL E MÉTODO}

O presente estudo trata-se de uma revisão da literatura, com artigos em inglês e português, indexados nas bases de dados MedLine, Scielo e Lilacs. Para a busca dos artigos foram utilizados os seguintes descritores: coagulopatias hereditárias (hereditary coagulopathies), hemofilia (hemophilia) e doença de Von Willebrand (Von Willebrand disease).

\section{RESULTADOS E DISCUSSÃO}

As alterações apresentadas nas coagulopatias são expressas com herança genética, quadro clinico e laboratorial variáveis. Etiologicamente, essas doenças hereditárias podem ser classificadas de acordo com as seguintes deficiências dos seguintes fatores: fibrinogênio, protrombina, von Willebrand e fatores V, VII, VIII, IX, X, XI, XII E XIII, sendo a deficiência do fator VIII ligada à hemofilia tipo A e a do fator IX à hemofilia tipo $\mathrm{B}^{4,6}$.

\section{- EPIDEMIOLOGIA}

A hemofilia tende a acontecer numa frequência estimada de aproximadamente um em cada $10 \quad 000$ nascimentos, logicamente em países em que as ferramentas de diagnóstico estão prontamente disponíveis ${ }^{7,8}$. Em termos gerais, essa doença acomete aproximadamente 400000 no mundo ${ }^{7}$, sendo a hemofilia A $(80-85 \%)$ mais comum que a hemofilia $\mathrm{B}^{8}$.

Em 2011 foi publicada a mais recente edição do Hemophilia's Annual Global Survey, esse levantamento inclui dados sobre mais de 265 mil pessoas com hemofilia, doença de von Willebrand e outras doenças hemorrágicas em 108 países $^{7}$.

No Brasil, o levantamento mais recente constatou que há um número de 16.076 pacientes com coagulopatias, dos quais $8.369(52,06 \%)$ correspondem à hemofilia $\mathrm{A}$; $1.609(10,01 \%)$, à hemofilia $\mathrm{B} ; 4.451(27,69 \%)$, à doença de von Willebrand; e $1.647(10,25 \%)$, a outras coagulopatias hereditárias e aos demais transtornos hemorrágicos ${ }^{9}$.

Regionalmente a distribuição percentual das coagulopatias hereditárias por diagnóstico segue a ordem da região Sudeste concentrando aproximadamente $50 \%$ dos casos de coagulopatias do Brasil, acompanhada pela Região Nordeste $(20,9 \%)$, Sul (16,9\%), Centro-Oeste $(6,71 \%)$ e Norte $(6,62 \%)$. O Estado da Paraíba concentra pouco mais de $1 \%$ da estatística nacional ${ }^{9}$.

Considerando-se o sexo como variável nos diversos tipos de coagulopatias hereditárias e à doença de von Willebrand, nas hemofilias A e B, aproximadamente 97\% dos pacientes são do sexo masculino e cerca de $3 \%$ são do sexo feminino. A maior parte deste percentual provavelmente representa portadoras de hemofilia com baixo nível de fator VIII ou IX. Esse contexto se altera quando consideramos a doença de von Willebrand, na qual a maior parte dos acometidos pertencem ao sexo feminino ${ }^{9}$.

No que se refere à análise dos pacientes por faixa etária, a maior prevalência ocorreu na faixa etária dos 20-29 anos, que corresponde a $23,17 \%$ dos casos 9 .

\section{○ HEMOFILIAS}

As hemofilias são doenças hemorrágicas de origem adquirida ou hereditária. A forma adquirida é mais rara, e caracteriza-se pela produção de anticorpos contra os fatores de coagulação, podendo estar associada a doenças ou ser de origem idiopática $^{10,7}$. Já a congênita é uma doença genética recessiva ligada ao sexo, resultante de mutações nos genes que codificam os fatores de coagulação ${ }^{8}$. Quando o defeito se localizar no mecanismo de produção do fator VIII chamar-se-á hemofilia A (hemofilia clássica) ${ }^{6,11}$; se do fator IX, hemofilia B (doença de Christmas) $)^{11}$.

Essas alterações quando de caráter congênito, são transmitidas através do braço longo do cromossomo X, que carrega os genes responsáveis pela codificação dos fatores VIII e IX. Devido à recessividade do cromossomo X, elas são bastante incomuns em mulheres, sendo mais expressa em homens. As mulheres geralmente são apenas portadoras, podendo apresentar baixos níveis de fator relacionado à inativação do cromossomo X "normal",4,10.

Do ponto de vista genético, sua transmissão ocorre quase que exclusivamente a indivíduos do sexo masculino por mãe portadora, porém, em uma parcela significativa dos casos, a doença pode origina-se a partir de mutação nova. Em casos raros, a hemofilia pode acontecer em mulher, decorrente da união de homem com hemofilia e mulher portadora $^{6}$

As principais manifestações clínicas das desordens hemorrágicas são os sangramentos, que podem ocorrer de forma espontânea ou induzida por trauma ou cirurgia ${ }^{2}$. Nessa perspectiva, pequenos procedimentos invasivos podem precipitar um episódio de sangramento prolongado, que além de ser doloroso para o paciente, também impede a conclusão do procedimento e compromete a cura da ferida ${ }^{3}$.

A pesquisa da história do paciente é muito importante, principalmente quando envolve sangramento excessivo após extração dentária ou pequenos cortes, equimoses espontâneas ou sangramento muscular; e transfusões de derivados de sangue. Quando existe um episódio hemorrágico prévio, tem-se uma probabilidade de $40 \%$ de existir alguma alteração ${ }^{12,13}$.

Alguns sinais e sintomas direcionam o diagnóstico, permitindo uma caracterização clínica do tipo de distúrbio. Por exemplo: hematomas musculares ou hemorragias intraarticulares (decorrentes de distúrbios da hemostasia primaria que atinge vasos e plaquetas) são indicativos de hemofilias. Já hemorragias na pele e nas mucosas causados possivelmente por alterações da hemostasia primaria que envolve a cascata de coagulação pode indicar diagnóstico de doença de von Willebrand ${ }^{12}$.

O grau, a localização, a duração do dano e a idade do paciente têm impacto sobre a gravidade da hemorragia e sobre as complicações encontradas. Atualmente o padrão de gravidade da hemofilia tem sido definido como pelo Scientific and Standardization Committee of the International Society on Thrombosis and Haemostasis da seguinte forma: leve, quando há hemorragia grave decorrente de traumas maiores ou cirurgias; moderada, quando existe presença de sangramentos espontâneos ocasionais; e grave, em sangramentos espontâneos predominantes em articulações e músculos ${ }^{14}$.

Clinicamente na forma grave observam-se sangramentos espontâneos ou desencadeados por pequenos traumas, sendo esta manifestação dolorosa, incapacitante e deformante. $\mathrm{Na}$ moderada, os sangramentos ocorrem geralmente associados a pequenos traumas, porém com uma menor frequência. Já na hemofilia leve, não há sangramentos espontâneos, neste tipo hemorragias só ocorrem quando houver trauma intenso, cirurgia, biopsia; sendo muitas vezes reconhecida apenas no adulto em casos de cirurgia ou extração dentaria ${ }^{15}$.

As hemartroses são as manifestações hemorrágicas mais características das formas graves da doença, atingindo mais frequentemente as articulações do joelho, tornozelo, cotovelo, ombro e coxo femoral. Essas alterações articulares 
geralmente geram um processo degenerativo caracterizado por sinovite crônica, anquilose, deformidade articular e alto índice de invalidez permanente nesses pacientes ${ }^{6}$.

\section{- DOENÇA DE VON WILLEBRAND}

E uma doença hemorrágica resultante de defeito quanti e/ou qualitativo do fator von Willebrand (FvW). É herdada como caráter autossômico dominante, graças a mutações no gene localizado no braço curto do cromossomo $12^{4,3}$. Caracteriza por ser uma doença com disfunção plaquetária, com prejuízo na formação do FvW, além de ser acompanhada por diminuição dos níveis do fator VIII coagulante $^{16}$.

O FvW é uma glicoproteína produzida pelas células endoteliais e megacariócitos, presente no plasma e nas plaquetas. Ele tem duas funções principais: (1) de formar a ponte que permite a aderência das plaquetas na superfície endotelial danificada, promovendo a formação do tampão plaquetário no local da lesão endotelial e (2) a de ligar e transportar o fator VIII (FVIII), protegendo-o da degradação proteolítica no plasma tornando-o mais estável ${ }^{4,3,17}$.

Classicamente, a DvW apresenta-se com tempo de sangramento (TS) alargado com número de plaquetas normais. A maioria dos casos se apresenta na forma leve e os pacientes podem apresentar exames de rotina da coagulação normais ou discretamente alterados ${ }^{3,16}$.

Essa doença afeta ambos os sexos, podendo se expressar na forma de padrões de sangramento espontâneo ou provocado. Os indivíduos com doença de von Willebrand possuem uma tendência para hematomas fáceis, epistaxes frequentes e menorragia ${ }^{16,5}$.

Existem mais de 21 subtipos distintos da DVW, entretanto a classificação atualmente utilizada, proposta por Evan Sadler, consiste em sete diferentes subtipos, sendo os três mais comuns descritos a seguir: tipo I é a mais comum (70\%) e apresenta caráter autossômico dominante, sendo considerada como um defeito quantitativo. Ele geralmente é confirmado com a combinação de anormalidades de plaquetas testes de função uma diminuição no fator von Willebrand, assim como também a diminuição proporcional da atividade do fator VIII. O tipo II, por sua vez, tem quatro subtipos (A, B, M, N), todos os quais são defeitos qualitativos e geralmente todos são dominantes autossômicos e o tipo III é raro, mas muito grave, caracterizado pela herança autossômica recessiva e assemelha-se, geralmente, a hemofilia $\mathrm{A}^{4,16,17}$.

\section{- DIAGNÓSTICO}

Devido à semelhança clinica entre as afecções hemorrágicas um diagnóstico correto é essencial para garantir um tratamento adequado. Para tanto, o clínico deve ter acesso à história clínica do paciente e de exames laboratoriais adequados ${ }^{6}$. Nessa perspectiva, o diagnóstico de DvW é geralmente realizado em 3 etapas: (1) identificação do paciente com possível DvW, baseado na história clínica e em testes laboratoriais; (2) diagnóstico e definição do tipo de DvW e (3) caracterização do subtipo da doença $^{18}$.

Os testes laboratoriais necessários para o diagnóstico da DvW são divididos em: testes de triagem: Tempo de sangramento (TS), contagem de plaquetas e TTPA; testes confirmatórios: Determinação do fator VIII:C, determinação plasmática do $\mathrm{FvW}$ antígeno ( $\mathrm{FvW}: \mathrm{Ag}$ ), determinação da atividade do $\mathrm{FvW}$ (FvW:RCo), ligação do $\mathrm{FvW}$ ao Colágeno (FvW:CB) e testes especiais: Agregação plaquetária induzida pela ristocetina (RIPA), capacidade de ligação ao FVIII (FvW:FVIII), análise multimérica do $\mathrm{FvW}^{18}$.
No caso das hemofilias, o diagnostico laboratorial consiste na realização dos testes TTPA (teste de triagem), determinação de FVIII: C e/ou FIX: $\mathrm{C}$ e pesquisa e quantificação do inibidor ${ }^{18}$.

\section{- TIPOS DE EXAMES DE AVALIAÇÃO DA} COAGULAÇÃO

A. Contagem de Plaquetas: Deve ser realizada para detectar trombocitopenia (contagem plaquetária inferior a $150.000 / \mathrm{mm} 3$ ), porém raramente ocorre sangramento até que a diminuição do número de plaquetas atinja $50-70.000 / \mathrm{mm}$. O valor normal médio é de aproximadamente $240.000 / \mathrm{mm}^{12,19,8}$.

B. Tempo de Sangramento segundo Duke (TS): É um teste laboratorial realizado in vivo que se refere à duração do sangramento de uma pequena incisão provocada com o auxílio de uma lanceta. O resultado normal é menor que três minutos. Quando esse tempo é prolongado, evidencia-se uma anormalidade plaquetária quantitativa ou qualitativa, um defeito na interação plaqueta-vaso (doença de von Willebrand) ou uma doença vascular primária ${ }^{12,20,21,8}$.

C. Tempo de Tromboplastina Parcial Ativado (TTPa): Exame que avalia deficiências ou inibidores dos fatores de coagulação nas vias intrínseca e comum da cascata da coagulação (pré-calicreína, cininogênio de alto peso molecular, fatores XII, XI, IX, VIII, X, V, protrombina e fibrinogênio). Normalmente este tempo é menor que 35 segundos, podendo variar entre 25 e 39 segundos ${ }^{12,20,8}$.

D. Tempo de Protrombina (TP): Esse exame avalia a reatividade dos fatores da via extrínseca e comum final. Anormalidades nos fatores VII, V, X, protrombina ou fibrinogênio, além da presença de inibidores podem prolongar o TP. O valor normal está situado entre 10 e 14 segundos, entretanto, é mais bem avaliado como uma porcentagem do tempo obtido pelo controle (atividade da protrombina) ${ }^{12,20,8}$.

E. Tempo de Trombina (TT): Este teste mede a velocidade de conversão de fibrinogênio em fibrina, última fase da coagulação, identificando a desfibrinogenemia. Os valores normais estão situados entre 9 e 12 segundos, esses são alterados quando os níveis de fibrinogênio estão abaixo de 70 a 100 mg/dL, ou quando existem níveis elevados de produtos de degradação da fibrina ou do fibrinogênio ${ }^{12}$.

\section{CONCLUSÃO}

É de suma importância os profissionais terem conhecimento acerca do conceito, fisiopatologia e, principalmente, do diagnóstico das coagulopatias hemorrágicas, uma vez que a semelhança da apresentação clínica dessas afecções pode dificultar esse diagnóstico, interferindo, assim, no estabelecimento de um tratamento. Apesar de esse estudo ter sido amplo, percebe-se a necessidade de evidências mais profundas acerca das coagulopatias hemorrágicas apresentadas.

\section{REFERÊNCIAS}

1. Douglas CR. Tratado de fisiologia aplicada às ciências médicas. 6 ed. Rio de Janeiro: Guanabara Koogan; 2006.

2. Guyton AC Hall JE. Tratado de Fisiologia Médica. 12 ed. Rio de Janeiro: Elsevier; 2011.

3. Israels S, Schwetz N, Boyar R, McNicol A. Bleeding Disorders: Characterization, Dental Considerations and Management. J Can Dent Assoc. 2006; 72(9):827-40. 
4. Brasil. Ministério da Saúde. Normas e Manuais Técnicos: Manual de tratamento das coagulopatias hereditárias. Série A. Brasília, Coordenação Geral de Documentação e Informação, 2006.

5. Marques RVCF, Conde DM, Lopes FF, Alves CMC. Atendimento odontológico em pacientes com Hemofilia e Doença de von Willebrand. Arq. Odontol. 2010; 46(3): 176-80.

6. Rezende SM. Distúrbios da hemostasia: doenças hemorrágicas. Rev Med Minas Gerais. 2010; 20(4): 53453 .

7. World Federation of Hemophilia (WFH). Report on the Annual Global Survey 2011. Canadá, December 2011. Disponível em: http://www1.wfh.org/ publications/ files/ pdf-1488.pdf. Acesso: 03 abr. 2016.

8. Srivastava A, Brewer AK, Mauser-Bunschoten EP, Key NS, Kitchen S, Ludlam CA, et. al. Guidelines for the management of hemophilia. Hemophilia. 2013; 19(1):147.

9. Brasil. Ministério da Saúde. Estatística e Informação em Saúde: Perfil das coagulopatias hereditárias no Brasil: 2009-2010. Série G. Brasília, Coordenação Geral de Sangue e Hemoderivados, 2012a.

10. Brasil. Ministério da Saúde. Normas e Manuais Técnicos: Manual de atendimento odontológico a pacientes com coagulopatias hereditárias. Série A. Brasília, Coordenação Geral de Documentação e Informação, 2008a.

11. Mazzoni A. Conduta odontológica para atendimento de pacientes hemofílicos. J APCD. [Periódico na Internet]. Disponível em: http://www.apcd.org.br/. Acesso: 03 abr. 2016.

12. Rizzatti EG; Franco RF. Investigação diagnóstica dos distúrbios hemorrágicos. Medicina, Ribeirão Preto. 2001; 34:238-247.

13. Wisniewski D, Kluthcovsky ACGC. O Perfil dos Pacientes Portadores de Coagulopatias de uma Região do Sul do Brasil. Cogitare Enferm. 2008; 13(2):212-9.

14. White GC 2nd, Rosendaal F, Aledort LM, Lusher JM, Rothschild C, Ingerslev $\mathrm{J}$, et al. Definitions in hemophilia. Recommendation of the scientific subcommittee on factor VIII and factor IX of the scientific and standardization committee of the International Society on Thrombosis and Haemostasis. Thromb Haemost. 2001; 85(3):560.

15. Ross J. Perspectivas de las portadoras de hemophilia. Montreal: Federación Mundial de Hemofilia, 2004. Serie monográfica El tratamiento de la hemofilia. Montreal, Canadá. Federación Mundial de Hemofilia, 2004.

16. Barbosa FT, Cunha RM, Barbosa LT. Doença de von Willebrand e Anestesia. Rev Bras Anestesiol. 2007; 57(3):315-23.

17. Dalati MH, Kudsi Z, Koussayer LT, Dalati MF, Mawla MF. Bleeding disorders seen in the dental practice. Dent Update. 2012; 39(4):266-8.

18. Brasil. Ministério da Saúde. Normas e Manuais Técnicos: Manual de diagnostico laboratorial das coagulopatias hereditárias e plaquetopatias. Série A. Brasília, Coordenação Geral de Documentação e Informação, 2012b.

19. Kriz N, Rinder CS, Rinder HM. Physiology of Hemostasis: With Relevance to Current and Future Laboratory Testing. Clin Lab Med. 2009; 29(2):159-74.

20. van Herrewegen F, Meijers JC, Peters M, van Ommen $\mathrm{CH}$. The bleeding child. Part II: Disorders of secondary hemostasis and fibrinolysis. Eur J Pediatr. 2012; 171(2):207-14.

21. van Ommen $\mathrm{CH}$, Peters M. The bleeding child. Part I: primary hemostatic disorders. Eur J Pediatr. 2012; 171(1):1-10.

\section{CONFLITO DE INTERESSES}

Os autores declaram não haver conflitos de interesse.

\section{AUTOR PARA CORRESPONDÊNCIA}

\section{Yago Tavares Pinheiro}

yagostavares5@gmail.com

Submetido em 25/02/2017

Aceito em 22/03/2017 